\title{
C. Vandermotten, B. Dézert, L'identité de l'Europe, histoire et géographie d'une quête d'unité
}

Paris : A. Colin, 2008

Michel Deshaies

\section{OpenEdition \\ Journals}

Édition électronique

URL : http://journals.openedition.org/rge/1619

DOI : 10.4000/rge.1619

ISSN : 2108-6478

Éditeur

Association des géographes de l'Est

Édition imprimée

Date de publication : 1 juin 2007

ISSN : 0035-3213

Référence électronique

Michel Deshaies, «C. Vandermotten, B. Dézert, L'identité de l'Europe, histoire et géographie d'une quête d'unité », Revue Géographique de l'Est [En ligne], vol. 47 / 3 | 2007, mis en ligne le 08 novembre 2011, consulté le 23 septembre 2020. URL : http://journals.openedition.org/rge/1619 ; DOI : https://doi.org/ $10.4000 /$ rge. 1619

Ce document a été généré automatiquement le 23 septembre 2020.

Tous droits réservés 
C. Vandermotten, B. Dézert, L'identité de l'Europe, histoire et géographie d'une quête d'unité

Paris : A. Colin, 2008

Michel Deshaies

\section{RÉFÉRENCE}

C. Vandermotten, B. Dézert, L'identité de l'Europe, histoire et géographie d'une quête d'unité, Paris : A. Colin, 2008, 334 p. 
1 L'ouvrage des professeurs Vandermotten et Dézert vient à point nommé à l'heure où la construction européenne traverse des difficultés croissantes liées au scepticisme, voire même à l'incompréhension des peuples. En effet, si les non exprimés par trois peuples (français, néerlandais, irlandais) aux référendums sur les projets de constitution européenne ont de nombreuses causes, ils sont notamment le fruit des doutes sur la place présente et plus encore future de leur pays en Europe.

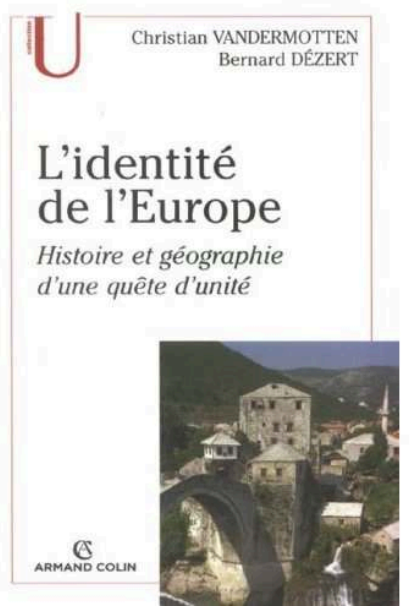
Or, cette inquiétude qui gagne une partie importante de l'opinion dans la plupart des États-membres n'est pas sans lien avec les changements politiques profonds qui, depuis 1989, ont bouleversé la carte de l'Europe, permettant la réunification du continent et l'élargissement de l'Union européenne. Face à ces changements, la crainte de beaucoup de peuples européens est de voir l'identité de leur nation se dissoudre dans un ensemble aux contours et à l'identité flous.

2 L'ouvrage de Vandermotten et Dézert apporte justement un éclairage essentiel à la question de l'identité européenne à travers une synthèse géographique particulièrement réussie. En effet, la démarche des auteurs est d'étudier la géographie de l'Europe en privilégiant les temps longs et en montrant l'importance des héritages dans la géographie actuelle d'un continent en quête d'unité. Il s'inscrit donc dans une optique de géographie historique qui est ici magistralement maîtrisée pour éclairer les enjeux contemporains de la construction européenne.

3 Sous un volume relativement modeste pour l'ampleur du sujet (334 p.), l'ouvrage rédigé dans une langue claire et précise, offre plus que des informations, une véritable culture géographique de l'Europe. Il est abondamment illustré de 75 cartes et figures et de près de 50 photographies dont on regrettera seulement qu'elles ne soient pas en couleur en raison de l'habituelle austérité des éditeurs français. En effet, les nombreuses photographies de paysages que les auteurs ont pris soin d'insérer perdent beaucoup et sont même parfois difficiles à interpréter en raison de reproductions en noir et blanc de qualité très médiocre. Les cartes sont par contre le plus souvent très claires, même si certaines auraient nécessité un format plus important.

4 L'ouvrage organisé en 8 chapitres présente d'abord les fondements géographiques et historiques du continent (chapitres 1 à 4), examine les mutations économiques, politiques et démographiques (chapitres 5 et 6) de la deuxième moitié du XXe s. et se termine par une régionalisation de l'espace européen (chapitre 7) et une vision prospective de l'évolution géographique de l'Europe reposant en partie sur une lecture critique du rapport ESPON.

5 La vision sur les temps longs de la géographie de l'Europe fait apparaître que la délimitation du continent est largement le produit de l'histoire et que ses contrastes internes, notamment la suprématie économique de l'Europe occidentale est ancienne, puisqu'elle est déjà affirmée au XVIe s. Il est ainsi montré le rôle qu'a joué l'urbanisation importante de l'Europe du Nord-Ouest dans le développement de la 
révolution industrielle; si bien que si la révolution industrielle représente une rupture économique et sociale majeure, elle ne bouscule pas fondamentalement la géographie préindustrielle puisqu'elle ne fait qu'accroître les écarts de développement entre l'Europe du Nord-Ouest et les périphéries méditerranéennes et orientales.

6 Ayant ainsi présenté les conditions de la formation de l'espace européen, les auteurs dressent un tableau de la grande diversité des paysages ruraux qui est à la fois l'expression des conditions naturelles et de l'extrême richesse des cultures. Ce chapitre qui s'inscrit dans une des grandes traditions de l'étude géographique, propose un véritable tour d'Europe montrant à la fois les héritages d'une histoire séculaire inscrits dans la mosaïque paysagère et les transformations résultant des changements économiques intervenus depuis la Seconde guerre mondiale. Cette approche fait transition avec le chapitre consacré aux mutations de l'espace économique et politique durant le dernier demi-siècle. Les auteurs montrent à quel point les mutations ont été radicales, en conséquence notamment d'un essor économique sans précédent à l'Ouest, progressivement réuni en Union européenne, des transformations induites par la collectivisation puis la dé-collectivisation à l'Est et de la réunification capitaliste de l'Europe après 1990. Cette Europe en mutation est confrontée à une faible dynamique démographique résultant d'un effondrement de la fécondité. Cette situation implique pour l'avenir de réfléchir aux choix politiques permettant d'adapter les systèmes sociaux et économiques à un vieillissement inéluctable de la population. La faible croissance naturelle, presque générale en Europe (certains pays comme l'Allemagne ou l'Italie étant même engagés dans un processus de décroissance naturelle), peut être en partie compensée par les bilans migratoires qui différencient clairement deux Europe, celle de l'Ouest qui continue à attirer les migrants et celle de l'Est dont la décroissance naturelle est aggravée par les pertes migratoires.

7 Les grands traits hérités de la longue gestation de l'espace européen, plus ou moins profondément transformé par les mutations du dernier demi-siècle, se traduisent par des contrastes régionaux marqués qui permettent de distinguer six grands ensembles en fonction d'un modèle centre-périphérie. Rompant avec le schéma de la "Banane bleue " de R. Brunet, les auteurs proposent un découpage tenant plus compte des facteurs culturels et de certaines caractéristiques économiques en individualisant un " noyau ouest-européen » centré sur l'Allemagne rhénane et les Pays-Bas, englobant l'île de France par l'axe de la basse Seine et s'étendant d'un côté jusqu'aux Midlands britanniques, de l'autre jusqu'à Vienne et aux Alpes ainsi qu'à la région lyonnaise. Ce noyau ouest-européen qui s'individualise à la fois par ses fortes densités de population, sa richesse, la concentration des activités de commandement économique et financier, ainsi que son insertion dans les réseaux internationaux, est bordé au nord comme au sud par des régions à plus faibles densités, où les niveaux de produit par habitant sont moins importants en raison d'une économie à niveau d'intensité technologique plus faible. Il s'agit de la plus grande partie du territoire français à l'exception des contrées méditerranéennes, de l'Allemagne du Nord et de l'Est, ainsi que des périphéries britanniques, y compris l'Irlande. Deux aires périphériques aux caractéristiques bien différentes s'individualisent ensuite au nord et au sud de ce noyau central européen. Il s'agit en premier lieu des pays nordiques qui figurent parmi les régions ayant atteint le niveau de développement le plus élevé, malgré la marginalité croissante des contrées septentrionales et en second lieu des pays méditerranéens, dont on peut toutefois douter de la pertinence à les rassembler dans une "aire méditerranéenne ». En effet, comme le soulignent d'ailleurs les auteurs, on y trouve à la fois une grande partie des 
régions périphériques rurales pauvres et marquées par l'archaïsme de l'Union européenne et l'une des régions les plus riches et les plus développées que constitue l'Italie du Nord. Mais, tout en rappelant que cette région présente bien des points communs avec le noyau central ouest-européen, elle s'en différencie par les caractéristiques du capitalisme industriel, plus familial et moins intensif en capital que celui de l'Europe rhénane. Enfin l'Europe centre-orientale et balkanique et l'Europe orientale (l'ancienne URSS à l'exclusion des Pays baltes) constituent les deux autres ensembles régionaux subdivisés en sous-ensembles en fonction de leurs niveaux d'intégration à l'économie européenne occidentale. Dans tous ces pays, les contrastes sont forts et s'accentuent entre quelques capitales assez bien intégrées aux réseaux internationaux et des territoires marqués soit par l'archaïsme et l'insuffisance des infrastructures, soit par les difficultés de reconversion des régions d'industrialisation lourde héritées du système communiste.

8 Le dernier chapitre propose des perspectives à moyen terme pour l'évolution de la géographie de l'Europe qui, suivant les choix retenus en matière de politique de cohésion, verra un renforcement des contrastes et notamment de l'avantage du noyau central européen, ou au contraire étendra l'espace de développement, en réduisant les risques de marginalisation des régions périphériques. L'Europe doit aussi affronter les grands défis que constituent la sécurisation de son approvisionnement énergétique, de plus en plus dépendant de régions extra-européennes, les problématiques environnementales liées à la consommation énergétique et le maintien de sa place dans le monde. Les réponses à apporter sont largement dépendantes de l'avenir de la construction européenne qui nécessitera probablement une remise à plat du fonctionnement des institutions ; ce qui paraît plus que jamais impératif pour sortir l'Europe de l'immobilisme actuel.

On le voit, ce livre sur "l'identité de l'Europe » ne peut que retenir l'attention, non seulement des géographes et des historiens, mais plus largement des citoyens curieux de mieux connaître l'Europe dont on peut dire sans conteste qu'elle souffre d'un manque d'image.

\section{AUTEURS}

\section{MICHEL DESHAIES}

CERPA, Université Nancy 2 